\title{
Influence of Nonminimum Phase Zeros on the Performance of Optimal Continuous-Time Iterative Learning Control
}

\author{
David H. Owens, Bing Chu, Eric Rogers, Chris T. Freeman, and Paul L. Lewin
}

\begin{abstract}
Iterative learning control can be applied to systems that execute the same tracking task over a finite time duration. An execution is known as a trial, and once each is complete, the system resets to the starting location and the next trial begins. All previous trial information is available for use in constructing the control input for the next trial, and the basic idea is to improve tracking performance from trial-to-trial. This brief analyzes the effects of nonminimum phase zeros on the trial-to-trial error norm convergence of norm optimal iterative learning control, a commonly used algorithm, for differential linear systems with supporting experimental results from a test facility.
\end{abstract}

Index Terms-Experimental verification, iterative learning control, linear nonminimum phase systems.

\section{INTRODUCTION}

$\mathbf{M}$ ANY industrial systems repeat the same operation over and over again on a finite duration. An example is a gantry robot undertaking a pick and place operation in synchronization with a conveyor system, where the sequence is: 1) collect an object from a fixed location; 2) transfer it over a finite duration; 3) place the object on the moving conveyor; 4) return to the original location for the next object; and 5) repeat the previous four steps indefinitely. Each execution of the operation is known as a trial and iterative learning control (ILC) uses information from previous trials, available once they are complete, to update the control input to be applied on the next trial. Reviewing the available literature, starting from the surveys briefs [1], [2], shows that ILC is applied to an extremely broad range of application domains, including industrial robotics, laser installations, and aerodynamic control systems.

A significant number of ILC algorithms are gradient based, i.e., they aim to reduce the error norm through the reduction of a cost function. One member of this class is termed norm optimal ILC (NOILC) where the cost function is quadratic in the current trial error and in the difference between the control input vector on two successive trials. Desirable properties of NOILC include a guarantee of trial-to-trial convergence of

Manuscript received July 23, 2012; revised April 5, 2013; accepted June 8, 2013. Manuscript received in final form June 8, 2013. Recommended by Associate Editor T. Zhang.

D. H. Owens is with Electronics and Computer Science, University of Southampton, Southampton SO17 1BJ, U.K., and also with the Department of Automatic Control and Systems Engineering, University of Sheffield, Sheffield S1 3JD, U.K. (e-mail: d.h.owens@sheffield.ac.uk).

B. Chu, E. Rogers, C. T. Freeman, and P. L. Lewin are with Electronics and Computer Science, University of Southampton, Southampton SO17 1BJ, U.K (e-mail: b.chu@ecs.soton.ac.uk; etar@ecs.soton.ac.uk; cf@ecs.soton.ac.uk; pll@ecs.soton.ac.uk).

Color versions of one or more of the figures in this paper are available online at http://ieeexplore.iee.org.

Digital Object Identifier 10.1109/TCST.2013.2267743 the error, measured by a suitable norm, to zero, with some control over the convergence rate through stipulation of the weights in the cost function. The aim is to achieve trial-bytrial error reduction without allowing excessive changes in the control input. More recent contributions until those listed in the survey briefs [1], [2] include [3]-[5] highlighting the continued interest in this design method.

An ILC algorithm seeks to construct an inverse of the plant dynamics over multiple trials, and hence the issue of nonminimum phase (NMP) zeros has been a key challenge for many years, with common approaches based on stable inversion and pseudo-inverse-based techniques [6], [7]. In common with classical feedback control, ILC is limited in what it can achieve for a given plant model structure, and NMP dynamics are found to present considerable performance difficulties. NMP zeros are commonly encountered in both discrete and continuous-time control system design, with physical examples including sway control of ships and heave control of submarines, with aircraft exhibiting equivalent types of motion to both of these phenomena.

Nonminimum phase plant zeros do have a very significant influence on the trial-to-trial convergence of NOILC. This is shown for the direct digital control case in [8] where the analysis is based on the theory of bounded linear operators mapping a finite-dimensional vector space into itself. These results do not extend to the continuous-time systems where the vector spaces are infinite-dimensional. This is a significant short-coming, even in the absence of NMP zeros in the plant transfer-function, especially if design by emulation, as opposed to direct digital control, is the only possible, or preferred, design method as the signals involved are in continuous-time. An example of NOILC design by emulation that is applied to a physical example with a minimum phase transfer-function model is briefly discussed and referenced in Section II. In addition, no experimental results are available to inform and support the analysis of NOILC for NMP plants, a necessary requirement before control design, and it is this area that is addressed in this brief.

After a summary of the required background results, a simulation example is given to illustrate that NOILC can exhibit slow convergence behavior for systems with NMP plant zeros, and this is subsequently shown to depend on the zero locations, the chosen reference vector and the error on the first trial. Slow convergence is a problem in practice as each trial is a physical experiment and the number of experiments that can be undertaken in reasonable time and at acceptable cost is inevitably limited. Also in many applications, the major performance issue is to maximize throughput (for example, placing objects onto a moving conveyor under synchroniza- 
tion) with high accuracy. Hence, there is a need to predict the effects of NMP zeros before commissioning a system, and it is in this area that the major new applications-oriented contributions of this brief arise. The theory is supported by experimental results from an electromechanical NMP system and Section II gives the required background.

\section{BACKGROUND}

Controllable and observable single-input, single-output systems modeled by a linear continuous time-invariant statespace model are considered and written in the ILC setting as

$$
\begin{aligned}
& \dot{x}_{k}(t)=A x_{k}(t)+B u_{k}(t), \quad x_{k}(0)=x_{0} \\
& y_{k}(t)=C x_{k}(t), \quad 0 \leq t \leq \alpha<\infty .
\end{aligned}
$$

In application, the system operates over the finite trial duration $\alpha$, it then resets to $t=0$ and the next trial begins. The nonnegative integer $k$ is the trial number, $x_{k}(t)$ is the $n \times 1$ state vector on trial $k$, and $y_{k}(t)$ and $u_{k}(t)$ are the output and input, respectively, on this trial. In addition, if $r(t)$ is the supplied reference signal, then $e_{k}(t)=r(t)-y_{k}(t), 0 \leq t \leq \alpha$, is the error on this trial. Without loss of generality, it is assumed that the initial state is independent of the trial number and that $x_{0}$ is known.

In common, with many other systems theory problems, a large class of control design methods in ILC are based on choosing the current trial input to minimize a cost function. In NOILC, the cost function is composed of quadratic terms in the current trial error and control input and one form is

$$
\begin{aligned}
J_{k+1}\left(u_{k+1}\right)= & \int_{0}^{\alpha}\left\{e_{k+1}^{T}(t) Q e_{k+1}(t)\right. \\
& \left.+\left(u_{k+1}(t)-u_{k}(t)\right)^{T} R\left(u_{k+1}(t)-u_{k}(t)\right)\right\} d t
\end{aligned}
$$

where $Q>0$ and $R>0$ are real scalars.

As discussed in Section I, NOILC is a widely used algorithm in ILC that continues to attract much interest. A particular feature of the cost function (2) is the penalty on the difference $u_{k+1}(t)-u_{k}(t)$ in the control signal used on successive trials. This is practically motivated and based on the idea that the error should reduce from trial-to-trial without an excessive increase in the control action demanded.

Recent work [9] has seen the successful transfer of ILC algorithms from industrial robotics to robotic-assisted upper limb stroke rehabilitation, where the control input on any trial is the electrical stimulation signal applied to assist the patient in performing a prescribed task, such as reaching out to grasp a cup. The goal is to promote patients' relearning of lost function and if progress is being made then their voluntary effort should go up and the level of assistive stimulation down from trial-totrial. This feature is found in patient trials and this is an ILC application area where the control input must be very tightly regulated, that is, the input cannot change significantly from trial-to-trial, and the input norm must be limited in addition to the error norm.

If all the entries in the state vector are available for measurement, the optimal input, see, for example [10], on trial $k+1$ is given by

$$
u_{k+1}(t)=u_{k}(t)-R^{-1} B^{T}\left[K(t) x_{k+1}(t)-\xi_{k+1}(t)\right]
$$
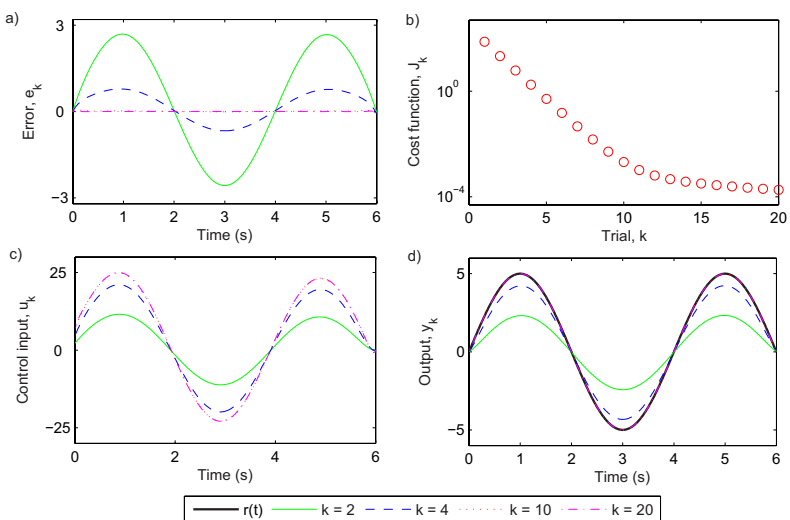

Fig. 1. NOILC performance from a representative minimum phase system. (a) Error, $e_{k}$. (b) Cost function, $J_{k}$. (c) Control input, $u_{k}$. (d) Output, $y_{k}$.

and takes the form of a state feedback control law defined by the feedback gain matrix $K(t)$ plus a predictive term $\xi_{k+1}(t)$, where these are calculated using

$$
\begin{aligned}
\dot{K}(t)= & -A^{T} K(t)-K(t) A+K(t) B R^{-1} B^{T} K(t) \\
& -C^{T} Q C, \quad K(\alpha)=0
\end{aligned}
$$

and

$$
\begin{aligned}
\dot{\xi}_{k+1}(t)= & -\left(A-B R^{-1} B^{T} K(t)\right)^{T} \xi_{k+1}(t) \\
& -C^{T} Q e_{k}(t), \quad \xi_{k+1}(\alpha)=0
\end{aligned}
$$

respectively. This causal algorithm is developed by considering the cost function as a linear quadratic tracking problem [that of combined tracking of $r(t)$ and disturbance accommodation, regarding $u_{k}(t)$ as a known disturbance on trial $\left.k+1\right]$. In application, the computation of $K(t)$ can be undertaken once before the trials commence and the predictive term can be constructed by reverse time simulations in the resetting time between trials.

Free-electron lasers comprise a major application area in which NOILC is applied to a physical application [12]. Here, models of the continuous-time dynamics are first constructed, NOILC designed using the algorithm given earlier and the resulting control law sampled and applied. The transferfunctions in this application area are minimum phase but the performance of NOILC is radically different if an NMP system is encountered, as illustrated next by a simulation example.

Consider the two transfer-functions

$$
G_{1}(s)=\frac{s+1}{(s+2)(s+3)}
$$

and

$$
G_{2}(s)=\frac{s-1}{(s+2)(s+3)}
$$

and zero initial conditions. The results of applying NOILC to the minimum-phase transfer-function $G_{1}(s)$ with $r(t)=$ $5 \sin (\pi / 2 t), Q=20, R=1$ are shown in Fig. 1 . The performance index $J$ reduces by several orders of magnitude over the first 10 trials, confirming rapid reduction in the error norm to a very small value.

The results for the NMP transfer-function $G_{2}(s)$ are shown in Fig. 2 for the same reference signal and cost function 

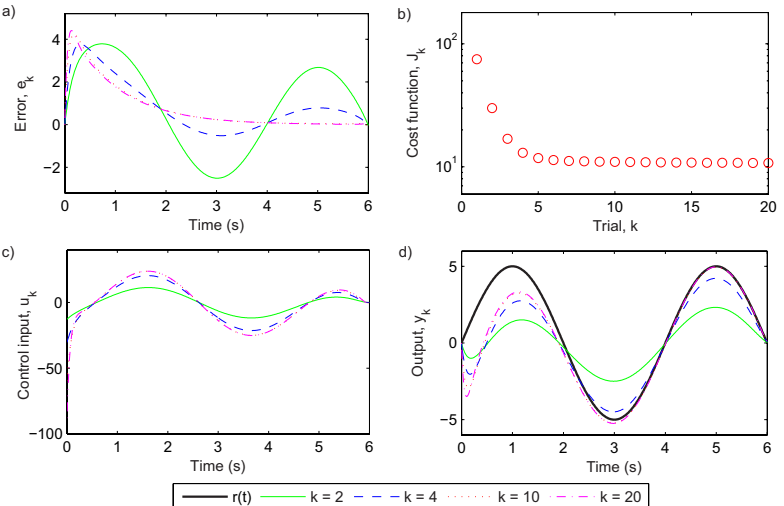

Fig. 2. Nonminimum phase example with apparent convergence to a nonzero, large error. (a) Error, $e_{k}$. (b) Cost function, $J_{k}$. (c) Control input, $u_{k}$. (d) Output, $y_{k}$.

weightings. Convergence to zero error is still guaranteed but the convergence behavior is very different. The algorithm again reduces the errors rapidly in the first few trials but then stagnates, at a much larger error value, and then moves along a plateau of almost constant error norm with only very small changes from trial-to-trial. In practical terms, the input after the sixth trial barely changes and hence the error appears to be converging to a nonzero value. The problem is that the nonzero error achieved is only an improvement of a factor of $\sim 10$ in norm over the initial error norm, an entirely unsatisfactory outcome from an applications standpoint.

The remainder of this brief develops new theoretical results that explain why this phenomenon occurs, with supporting experimental results from an NMP electromechanical system.

\section{Convergence AnAlysis}

\section{A. Preliminaries}

The NOILC algorithm of Section II is a particular case arising from analysis in a general Hilbert space setting [13] and the main background results required start from the following definition.

Definition 1: Consider a dynamic system with input $u$ and output $y$. Let $\mathcal{Y}$ and $\mathcal{U}$ be the output and input function spaces, respectively, and let $r \in \mathcal{Y}$ be a desired reference trajectory for the system. An ILC algorithm is successful if and only if it constructs a sequence of control inputs $\left\{u_{k}\right\}_{k \geq 0}$, which, when applied to the system, under identical experimental conditions, produces an output sequence $\left\{y_{k}\right\}_{k \geq 0}$ with the following properties of convergent learning

$$
\lim _{k \rightarrow \infty} y_{k}=r, \lim _{k \rightarrow \infty} u_{k}=u_{\infty} \in \mathcal{U}
$$

expressed in terms of the topologies assumed in $\mathcal{Y}$ and $\mathcal{U}$, respectively.

Let $\mathcal{Y}$ and $\mathcal{U}$, respectively, be real Hilbert spaces with corresponding inner products and norms denoted by $\langle\cdot, \cdot\rangle$ and $\|\cdot\|^{2}=\langle\cdot, \cdot\rangle$, respectively. When required, $\|x\|_{\mathcal{Y}}$, for example, is used to denote the norm of $x \in \mathcal{Y}$.

The linear system dynamics in operator form are given by $y=G u+d$, where $G: \mathcal{U} \rightarrow \mathcal{Y}$ is the system input/output operator. In addition, $G$ is assumed to be bounded with dense range, and $d$ describes initial conditions plus known trial independent disturbances. If $r \in \mathcal{Y}$ is the reference output or signal, the tracking error is $e=r-y=r-G u-d$. Systems described by (1) and (2) are a special case of this general problem setting when $\mathcal{U}$ and $\mathcal{Y}$, respectively, are taken as real vector spaces $L_{2}[0, \alpha]$ with inner products

$$
\begin{aligned}
& <y, z>\mathcal{Y}=\int_{0}^{\alpha} y^{T}(t) Q z(t) d t \\
& <u, v>\mathcal{U}=\int_{0}^{\alpha} u^{T}(t) R v(t) d t
\end{aligned}
$$

where $Q>0$ and $R>0$ are real scalars from the cost function (2) and $G: \mathcal{U} \rightarrow \mathcal{Y}$ is defined by

$$
\begin{aligned}
(G u)(t) & =\int_{0}^{t} C e^{A(t-\tau)} B u(\tau) d \tau \\
d(t) & =C e^{A t} x(0)
\end{aligned}
$$

Extensions to this problem are discussed in Section VI.

The NOILC problem can now be formulated as calculating, on completion of trial $k$, the control input on trial $k+1$ as the solution of the minimum norm optimization problem

$$
\begin{gathered}
u_{k+1}=\arg \min _{u_{k+1}}\left\{J_{k+1}\left(u_{k+1}\right): e_{k+1}=r-y_{k+1}\right. \\
\left.y_{k+1}=G u_{k+1}+d\right\}
\end{gathered}
$$

with performance index

$$
J_{k+1}\left(u_{k+1}\right)=\left\|e_{k+1}\right\|_{\mathcal{Y}}^{2}+\left\|u_{k+1}-u_{k}\right\|_{\mathcal{U}}^{2}
$$

The initial control $u_{0} \in \mathcal{U}$ can take any admissible value but, in application, will be a good first guess at the solution.

Standard Hilbert space techniques can now be used to show, one derivation is in [10], that this NOLIC problem has trial-to-trial error convergence in norm to zero tracking error independent of the plant dynamics $G$ with monotonically decreasing error norm

$$
\left\|e_{k+1}\right\| \mathcal{Y} \leq\left\|e_{k}\right\|_{\mathcal{Y}} \forall k \geq 0 \quad \lim _{k \rightarrow \infty}\left\|e_{k}\right\| \mathcal{Y}=0
$$

Also the trial-to-trial error and control input on trial $k+1$ are given for all $k \geq 0$ by

$$
e_{k+1}=\left(I+G G^{*}\right)^{-1} e_{k}, \quad u_{k+1}=u_{k}+G^{*}\left(I+G G^{*}\right)^{-1} e_{k}
$$

respectively, where the adjoint operator $G^{*}: \mathcal{Y} \rightarrow \mathcal{U}$ of $G$ is bounded [11].

An explicit representation of adjoint operators when the input and output vector spaces are $L_{2}[0, \alpha]$ Hilbert spaces with inner products $<u_{1}, u_{2}>\mathcal{U}$ and $<y_{1}, y_{2}>\mathcal{Y}$, respectively, defined by (6) is required in the analysis to follow. The following result provides such a characterization of the adjoint operator corresponding to a linear operator $L: \mathcal{U} \rightarrow \mathcal{Y}$ described by a state-space model.

Theorem 1: The adjoint operator $L^{*}$ of the linear map $L$ with $y=L u$ corresponding to the single-input, single-output linear time-invariant state-space model

$$
\dot{x}(t)=A_{0} x(t)+B_{0} u(t), \quad y(t)=C_{0} x(t)+D_{0} u(t)
$$

with $x(0)=0$, is the map $v=L^{*} z$ corresponding to the linear system

$\dot{p}(t)=-A_{0}^{T} p(t)-C_{0}^{T} Q z(t), v(t)=R^{-1}\left(B_{0}^{T} p(t)+D_{0} Q z(t)\right)$ 
with terminal boundary condition $p(\alpha)=0$.

The proof of this theorem requires the following.

1) The adjoint of the system $G$ generated by (1) is obtained by setting $A=A_{0}, B=B_{0}, C=C_{0}$, and $D_{0}=0$. Moreover, the addition of the term $D_{0} u(t)$ in the dynamics of $L$ is useful in subsequent analysis as the state-space model of the all-pass operator $G_{a}$ that arises subsequently in this brief has $D_{0}=1$.

2) If the system $L$ is associated with a transfer-function $L(s)=C_{0}\left(s I-A_{0}\right)^{-1} B_{0}+D_{0}$, then the adjoint system can be associated algebraically with the transfer-function $L^{*}(s)=R^{-1}\left(D_{0} Q-B_{0}^{T}\left(s I+A_{0}^{T}\right)^{-1} C_{0}^{T} Q\right)=\frac{Q}{R} L(-s)$.

Using the notation $\tilde{f}(t) \equiv f(\alpha-t)$ to denote the timereversed signal, it is straightforward to show that $\|f\|=$ $\|\tilde{f}\|$ and hence that

$$
v=L^{*} z \quad \Leftrightarrow \quad \tilde{v}=Q R^{-1} L \tilde{z} .
$$

That is, the response of $L^{*}$ to the input $z$ is just $Q R^{-1}$ times the time reversal of the response of $L$ to the timereversed $z$.

Proof: Let $z \in \mathcal{Y}$ and $u \in \mathcal{U}$. The result follows using the convolution solution of the state equations and a change of order of integration in the expression $\langle z, L u>\mathcal{y}$. Hence

$$
\left(L^{*} z\right)(t)=R^{-1}\left[B_{0}^{T} \int_{t}^{\alpha} e^{-A_{0}^{T}(t-s)} C_{0}^{T} Q z(s) d s+D_{0} Q z(t)\right]
$$

which has the state-space realization required.

\section{B. Analysis}

Suppose that the transfer-function $G(s)$ of the system (1) has $m$ zeros in the right-half of the complex plane denoted by $\left\{z_{j}: 1 \leq j \leq m_{0}\right\}$ with $z_{j}$ having multiplicity $n_{j}$ and $\sum_{j=1}^{m_{0}} n_{j}=m$. The starting point is the action of the operator $G^{*}$, and hence $G G^{*}$, on the linearly independent signals

$$
\theta_{j i}(t)=\frac{1}{(i-1) !} t^{i-1} e^{-z_{j} t}=\mathcal{L}^{-1}\left[\frac{1}{\left(s+z_{j}\right)^{i}}\right]
$$

$1 \leq i \leq n_{j}, 1 \leq j \leq m_{0}$, defined in terms of the plant zeros and their multiplicities. The zeros can be real or complex, but in this brief, attention is restricted to real zeros as the case of complex zeros follows with routine modifications as discussed after the next result characterizing the asymptotic behaviors of $G^{*} \theta_{j i}$ and $G G^{*} \theta_{j i}$ as $\alpha \rightarrow \infty$.

Theorem 2: Suppose that the system (1) is asymptotically stable and only has simple zeros of multiplicity $n_{j}=1$. Then

$$
\left(G^{*} \theta_{j}\right)(t)=e^{-z_{j} \alpha} Q R^{-1}\left(\tilde{y}_{z_{j}}\right)(t), \quad 1 \leq j \leq m
$$

where $y_{z_{j}}$ is defined as the response of $G$ with zero initial conditions to the input signal $e^{z_{j} t}$, that is

$$
y_{z_{j}}(t)=\mathcal{L}^{-1}\left[G(s) \frac{1}{s-z_{j}}\right] .
$$

Hence

$$
\lim _{\alpha \rightarrow \infty}\left\|G^{*} \theta_{j}\right\|=0, \lim _{\alpha \rightarrow \infty}\left\|G G^{*} \theta_{j}\right\|=0, \quad 1 \leq j \leq m .
$$

Proof: This follows from

$$
\left(G^{*} \theta\right)(t)=Q R^{-1} \widetilde{(G \tilde{\theta})}(t)=Q R^{-1} e^{-z_{j} \alpha} \tilde{y}_{z_{j}}(t)
$$

and hence

$$
\begin{aligned}
\left\|G^{*} \theta\right\| & =Q R^{-1}\|\widetilde{(\widetilde{G})}\| \\
& =Q R^{-1} e^{-z_{j} \alpha}\left\|\tilde{y}_{z_{j}}\right\| \\
& =Q R^{-1} e^{-z_{j} \alpha}\left\|y_{z_{j}}\right\| .
\end{aligned}
$$

Similarly

$$
\begin{aligned}
\left\|G G^{*} \theta\right\| & =Q R^{-1} e^{-z_{j} \alpha}\left\|G \tilde{y}_{z_{j}}\right\| \\
& \leq Q R^{-1}\|G\|_{L_{2}[0, \alpha]} e^{-z_{j} \alpha}\left\|y_{z_{j}}\right\|
\end{aligned}
$$

and finally

$$
\sup _{\alpha>0}\left\|y_{z_{j}}\right\|_{L_{2}[0, \alpha]}<\infty
$$

and

$$
\sup _{\alpha>0}\|G\|_{L_{2}[0, \alpha]}<\infty
$$

as $G$ is asymptotically stable until pole-zero cancelation occurs in the construction of $y_{z_{j}}$ and $\operatorname{Re}\left[z_{j}\right]>0$ by definition. In particular, the pole-zero cancelation and hence boundedness only occurs if $z_{j}$ is a zero of $G(s)$.

The following are also relevant to this last result.

1) The result of Theorem 2 extends to complex zeros where the appropriate $\theta_{j}$ functions are taken as the real and imaginary parts of $e^{-z_{j} t}$.

2) Theorem 2 also extends to multiple NMP zeros with the function $\left\{\theta_{j}\right\}$ replaced by the set $\left\{\theta_{j i}\right\}$ in a natural way.

Suppose that $G$ is NMP and the time interval $\alpha$ is large in the sense that all NMP zeros satisfy $\alpha z_{j} \gg 1$. Then the change in error $e_{k+1}-e_{k}=-G G^{*} e_{k+1}$ due to the component of $e_{k+1}$ in the subspace $\mathcal{E}_{+}$spanned by $\theta_{j}, 1 \leq j \leq m$ is extremely small. Consequently, the error norm sequence $\left\{\left\|e_{k+1}\right\|\right\}_{k \geq 0}$, although monotonic in $k$, will ultimately plateau out in the way seen in the simulation examples of Section II and the experimental results given in Section V.

The result of Theorem 2 can be summarized in the form

$$
\lim _{\alpha \rightarrow \infty}\left\|G^{*} \theta\right\|=0 \Leftarrow \theta \in \mathcal{E}_{+} .
$$

That is, $\theta \in \mathcal{E}_{+}$is sufficient to produce the required observed property and hence the physical implications given earlier. The following theorem shows that the implication can be reversed for a wide range of functions of practical importance.

Theorem 3: Suppose that $G$ generated by the system (1) is asymptotically stable with $m \geq 1$ zeros in the open right-half of the complex plane. Let $\theta \in L_{2}[0, \infty)$ be $m$-times differentiable with derivatives in $L_{2}[0, \infty)$, and also exponentially bounded in the sense that there exists $M_{\theta}>0$ and $\lambda_{\theta}>0$ such that $|\theta(t)| \leq M_{\theta} e^{-\lambda_{\theta} t}, \forall t \geq 0$. Then

$$
\lim _{\alpha \rightarrow \infty}\left\|G^{*} \theta\right\|=0 \Rightarrow \theta \in \mathcal{E}_{+} .
$$

Proof: Let $\stackrel{\alpha}{G}$ be stable and without loss of generality take $Q=R=1$. Suppose that $x \in L_{2}[0, \infty)$, and denote $x_{\alpha}$ as the truncation of $x$ to $x_{\alpha} \in L_{2}[0, \alpha$,$] where x_{\alpha}$ will also be regarded as an element of $L_{2}[0, \infty)$ by setting its value as 
zero for $t>\alpha$. It is required to show that if a signal $f \in$ $L_{2}[0, \infty)$ is $m$-times differentiable in $L_{2}[0, \infty)$ and is also exponentially bounded, that is, there exists $M>0$ and $\lambda>0$ such that $|f(t)| \leq M e^{-\lambda t}, \forall t \geq 0$, then $\lim _{\alpha \rightarrow \infty}\left\|G^{*} f_{\alpha}\right\|=0$ $\Rightarrow f \in \mathcal{E}_{+}$.

An important property for the following analysis is the identification of $\mathcal{E}_{+}$as the kernel of the polynomial differential operator $n(d / d t)$ where

$$
n(s)=\prod_{j=1}^{m}\left(s+z_{j}\right)=\sum_{j=0}^{m} a_{j} s^{j} .
$$

Using this, $G$ can be written as $G=G_{a} G_{m}$, where $G_{m}$ is stable, minimum-phase, and relative degree $k^{*} \geq 1$ and $G_{a}$ is stable and all-pass with transfer-function $(-1)^{m} n(-s) / n(s)=$ $1-H(s)$ where $H$ is strictly proper and stable with poles $\left\{-z_{j}\right\}_{1 \leq j \leq m}$. Also, let $H$ have state-space realization $\left(A_{a}, B_{a}, C_{a}\right)$ and its stability implies the existence of an exponential bound $\left|C_{a} e^{A_{a} t} B_{a}\right| \leq H_{0} e^{-\epsilon t}, \forall t \geq 0$, and some $\epsilon>0$. Also $G_{a}^{*} f_{\alpha}$ can be written as

$$
\begin{aligned}
f(t)-\int_{t}^{\alpha} C_{a} e^{A_{a}(s-t)} B_{a} f(s) d s \rightarrow \hat{f}(t) \\
=f(t)-\int_{t}^{\infty} C_{a} e^{A_{a}(s-t)} B_{a} f(s) d s
\end{aligned}
$$

point-wise as $\alpha \rightarrow \infty$ since, with $M^{\prime}=M H_{0}$, the integral term exists with bound

$$
\begin{aligned}
\left|\int_{t}^{\infty} C_{a} e^{A_{a}(s-t)} B_{a} f(s) d s\right| & \leq M^{\prime} \int_{t}^{\infty} e^{-\epsilon(s-t)} e^{-\lambda s} d s \\
& =\frac{M^{\prime}}{\epsilon+\lambda} e^{-\lambda t}, \forall t \geq 0
\end{aligned}
$$

In fact, $\hat{f}$ is continuous on $[0, \infty)$ and exponentially bounded, that is, $\hat{f} \in L_{2}[0, \infty)$.

$$
\begin{aligned}
\left|\hat{f}(t)-\left(G_{a}^{*} f_{\alpha}\right)(t)\right| & =\left|\int_{\alpha}^{\infty} C_{a} e^{A_{a}(s-t)} B_{a} f(s) d s\right| \\
& \leq \frac{M^{\prime}}{\epsilon+\lambda} e^{-(\epsilon+\lambda) \alpha} e^{\epsilon t}, \forall t \in[0, \alpha]
\end{aligned}
$$

which has norm $O\left(e^{-\lambda \alpha}\right)$ in $L_{2}[0, \alpha]$ while, in $L_{2}[\alpha, \infty)$, $\hat{f}(t)-\left(G_{a}^{*} f_{\alpha}\right)(t) \equiv \hat{f}(t)$ has arbitrarily small norm $\sqrt{\int_{\alpha}^{\infty}\left|\hat{f}(t)-\left(G_{a}^{*} f_{\alpha}\right)(t)\right|^{2} d t}$ as $\alpha \rightarrow \infty$. It follows that $G_{a}^{*} f_{\alpha} \rightarrow \hat{f}$, as $\alpha \rightarrow \infty$, in the norm topology of $L_{2}[0, \infty)$. Also, let $x \in L_{2}[0, \infty)$ be arbitrary and nonzero and also

$$
0=\lim _{\alpha \rightarrow \infty}\left\langle x_{\alpha}, G^{*} f_{\alpha}\right\rangle=\lim _{\alpha \rightarrow \infty}\left\langle G_{m} x_{\alpha}, G_{a}^{*} f_{\alpha}\right\rangle=\left\langle G_{m} x, \hat{f}\right\rangle .
$$

Also, $G_{m}$ maps $L_{2}[0, \infty)$ into itself and has range equal to the set of $k^{*}$ times differentiable functions in $L_{2}[0, \infty)$ which is dense in $L_{2}[0, \infty)$. Hence, $\hat{f}=0$, and, for $m \geq$ $1,\left|s I-A_{a}\right| \equiv n(s)$ and hence $n\left(A_{a}\right)=0$, from the Cayley-Hamilton Theorem. In a similar manner to the earlier mentioned, $f(t) \equiv \int_{t}^{\infty} C_{a} e^{A_{a}(s-t)} B_{a} f(s) d s$ for $t \in[0, \infty)$ from which (using $f^{(j)}$ to denote the $j$ th derivative of $f$ ) multiplying by $(-1)^{\ell} a_{\ell}, 0 \leq \ell \leq m$, adding and using

$$
\begin{aligned}
n\left(A_{a}\right)=0 & \\
\sum_{\ell=0}^{m}(-1)^{\ell} a_{\ell} f^{(\ell)}(t)= & \sum_{j=0}^{m-1}(-1)^{j} f^{(j)}(t) \\
& \times \underbrace{\sum_{\ell=j+1}^{m} a_{\ell} C_{a} A_{a}^{\ell-1-j} B_{a}, \forall \ell \geq 0 .}
\end{aligned}
$$

Also, $(-1)^{m} n(-s) \equiv n(s) G_{a}(s)$ and expanding $G_{a}(s)$ about the point at infinity and equating coefficients of $s^{\ell}$ for $0 \leq$ $\ell \leq m$ shows that the under-braced term is equal to $a_{\ell}-$ $(-1)^{m+\ell} a_{\ell}$. Also, it is routine to show that $n(d / d t) f \equiv 0$ that is, $f \in \mathcal{E}_{+}$, and the proof is complete.

For the broad class of functions in this theorem, $m$-dimensional subspace $\mathcal{E}_{+} \subset \mathcal{Y}$ provides a complete description of the slow convergence behavior. Moreover, $\forall \alpha>0$ the set of $m$-times differentiable functions with values in $L_{2}[0, \alpha]$ is dense in $L_{2}[0, \alpha]$ which suggests the theorem holds for $\theta \in L_{2}[0, \infty)$. Furthermore, the maximal subspace of $L_{2}[0, \infty)$ (denoted $\mathcal{E}_{+}^{\max }$ ) whose truncations to $L_{2}[0, \alpha]$ satisfy $\lim _{\alpha \rightarrow \infty}\left\|G^{*} \theta\right\|_{\mathcal{U}_{c}}=0 \Leftrightarrow \theta \in \mathcal{E}_{+}^{\max }$ contains $\mathcal{E}_{+}$. This suggests that the predicted value of error norm on the plateau derived in Section IV using $\mathcal{E}_{+}$will be a lower bound for the plateau observed using $\mathcal{E}_{+}^{\max }$. The remainder of this brief will restrict attention to the class of functions in this theorem as it covers many of the signals encountered in applications. A proof for the general case is a topic for future research.

\section{Model for Slow Algorithm Convergence USING LINEAR CONSTRAINTS}

In this section, an approximate mathematical model of the slow convergence behavior of NOILC for an NMP system is developed under the assumption that the trial duration $[0, \alpha]$ is long enough in the sense that $z_{j} \alpha \gg 1$ for $1 \leq j \leq m$. The model will take the form of a modified algorithm with the same structure and properties as NOILC but an error that converges to a nonzero limit, $e_{\infty}$. The norm of $e_{\infty}$ will be closely associated with that observed when the real NOILC algorithm is moving along the plateau, as demonstrated for the system $G_{2}(s)$ in Fig. 2.

To construct the approximate model, use the identity $e_{k+1}-$ $e_{k}=-G G^{*} e_{k+1}$ and write the error signal space as $\mathcal{E}_{+} \bigoplus \mathcal{V}$, where $\mathcal{V}$ is a suitable complement. The choice of $\mathcal{V}=\mathcal{E}_{+}^{\perp}$ as the most appropriate complement to $\mathcal{E}_{+}$is a crucial construction in what follows and is motivated by the fact that $G G^{*}$ is self-adjoint and hence has orthogonal eigenspaces. It also maps the unit ball of $\mathcal{E}_{+}$into a ball centered on $\{0\}$ of very small magnitude if $\alpha$ is large. This can be interpreted as suggesting that $\mathcal{E}_{+}^{\perp}$ is almost invariant under these circumstances. As a consequence, it is concluded that $e_{k+1}-e_{k}$ can be assumed to evolve predominantly in the subspace $\mathcal{E}_{+}^{\perp}$.

Over finite trial horizons, this assumption remains valid but ultimately fails as the effects of small errors in the model necessarily accumulate to allow NOILC to converge to zero. The assumption is that the approximation is valid over a sufficiently large number of trials to permit the plateau characteristics of NOILC and its model to coincide. To provide additional 
support for this construction, suppose that a bounded linear operator $H$ is such that $H H^{*}$ has finite dimensional, and hence closed, kernel $S$, that is, it maps $S$ exactly into the point set $\{0\}$ and it is easily shown that $H H^{*} S^{\perp} \subset S^{\perp}$.

Assuming that $G G^{*} \mathcal{E}_{+}^{\perp} \subset \mathcal{E}_{+}^{\perp}$, the error sequence satisfies the following linear constraint(s) for a large number of trials:

$$
<e_{k+1}-e_{k}, \theta>=0, \quad \forall \theta \in \mathcal{E}_{+}, \quad \alpha \gg 0
$$

or, as the set of linear constraints

$$
<e_{k+1}-e_{k}, \theta_{j}>=0, \quad 1 \leq j \leq m
$$

and hence, using $e_{k+1}-e_{k}=-G\left(u_{k+1}-u_{k}\right)$

$$
\begin{aligned}
u_{k+1} \in \Omega_{k+1} & =\left\{u:<u-u_{k}, \psi_{j}>=0,1 \leq j \leq m\right\} \\
\psi_{j} & =G^{*} \theta_{j} .
\end{aligned}
$$

The introduction of these linear constraints is the mechanism used below to construct the model of algorithm evolution developed to explain and predict the behavior of NOILC applied to NMP plants.

Remark 1: The approximation will break down as the trials progress because, ultimately, the error goes to zero but the model is expected to be capable of providing a good approximation over initial and possibly large number of trials where the plateau/stagnation effect is seen in practice.

In the model of NOILC behavior when applied to an NMP plant developed below, the trial input $u_{k+1}$ is approximated by the solution of the constrained optimization problem

$$
\begin{aligned}
& u_{k+1}=\arg \min \left\{J_{k+1}=\left\|e_{k+1}\right\|^{2}+\left\|u_{k+1}-u_{k}\right\|^{2}\right\} \\
& u_{k+1} \in \Omega_{k+1},
\end{aligned}
$$

and in the limit, as $k \rightarrow \infty$, these solutions provides a good approximation to behavior on the plateau. This optimization problem is solved by Lagrange multiplier techniques, that is, by minimizing the Lagrangian

$$
J_{k+1}^{\lambda}=\frac{1}{2} J_{k+1}+\sum_{j=1}^{m} \lambda_{j}\left\langle\psi_{j}, u_{k+1}-u_{k}\right\rangle
$$

over all $u_{k+1} \in \mathcal{U}$ and all Lagrange multipliers scalars $\lambda_{j}$. The necessary condition for a minimum is that $J_{k+1}^{\lambda}$ is stationary at the optimal $u_{k+1}$ and $\lambda=\left[\lambda_{1}, \lambda_{2}, \ldots, \lambda_{m}\right]^{T}$. Moreover, the addition of these constraints retains all properties of the NOILC algorithm except that the error sequence $\left\{e_{k}\right\}_{k \geq 0}$ does not necessarily converge to zero.

The solution of this problem is given by the following theorem where the notation $P_{\epsilon}$ is used to denote the selfadjoint, orthogonal projection operator onto the span of $m$ linearly independent vectors $\epsilon_{1}, \ldots, \epsilon_{m}$. In particular,

$$
P_{\epsilon} x=\left[\epsilon_{1}, \epsilon_{2}, \ldots, \epsilon_{m}\right] M_{\epsilon}^{-1} \beta_{\epsilon}(x)
$$

where $M_{\epsilon}$ is the $m \times m$ symmetric, positive definite matrix with $(i, j)$ element $\left\langle\epsilon_{i}, \epsilon_{j}\right\rangle$, and $\beta_{\epsilon}(x)$ is the $m \times 1$ vector with $i$ th element $\left\langle\epsilon_{i}, x\right\rangle$.

Theorem 4: Suppose that $G$ has a dense range. Then the solution of the constrained NOILC algorithm (31) and (32) on trial $k+1$ is

$$
u_{k+1}-u_{k}=\left(I-P_{\psi}\right) G^{*} e_{k+1}
$$

and has the monotonicity property

$$
\left\|e_{k+1}\right\| \leq\left\|e_{k}\right\| \quad \forall k \geq 0 .
$$

In addition, if $e_{k}$ converges to $e_{\infty}$ in the norm topology

$$
e_{\infty}=\sum_{j=1}^{m} \gamma_{j} \theta_{j}, \quad \gamma=\left[\gamma_{1}, \ldots, \gamma_{m}\right]^{T}=M_{\theta}^{-1} \beta_{\theta}\left(e_{0}\right)
$$

is the orthogonal projection of the initial error $e_{0}$ onto $\mathcal{E}_{+}$and

$$
\left\|e_{\infty}\right\|^{2}=\beta_{\theta}\left(e_{0}\right)^{T} M_{\theta}^{-1} \beta_{\theta}\left(e_{0}\right) .
$$

Before the proof of this theorem, the following remark is relevant.

Remark 2: The dense range assumption is automatically satisfied for state-space models with $L_{2}[0, \alpha]$ input and output spaces as continuous functions are dense in such spaces, which can be regarded as the completion of the pre-Hilbert space of continuous functions with the same norm and inner product.

Proof: The choice $u=u_{k}$ is suboptimal and the monotonicity property is easily established. Next, the conditions for a stationary point of the Lagrangian consists of the constraint equations and, taking the Fréchet derivative of the Lagrangian with respect to $u_{k+1}$, the equalities

$$
u_{k+1}-u_{k}=G^{*} e_{k+1}-\sum_{j=1}^{m} \lambda_{j} \psi_{j} .
$$

Taking the inner product with $\psi_{i}$ and using the constraints gives

$$
0=<\psi_{i}, G^{*} e_{k+1}>-\sum_{j=1}^{m} \lambda_{j}<\psi_{i}, \psi_{j}>, \quad 1 \leq i \leq m
$$

or, equivalently, $0=\beta_{\psi}\left(G^{*} e_{k+1}\right)-M_{\psi} \lambda$ and hence

$$
u_{k+1}-u_{k}=G^{*} e_{k+1}-\sum_{j=1}^{m} \lambda_{j} \psi_{j}=\left(I-P_{\psi}\right) G^{*} e_{k+1} .
$$

Multiplying this last expression by $G$ gives $e_{k+1}-e_{k}=$ $-G\left(I-P_{\psi}\right) G^{*} e_{k+1}$, and, assuming that $e_{k} \rightarrow e_{\infty}$, gives $0=G\left(I-P_{\psi}\right) G^{*} e_{\infty}$ or, as $G$ is injective

$$
G^{*} e_{\infty}=P_{\psi} G^{*} e_{\infty} \in \operatorname{span}\left\{\psi_{j}\right\}_{1 \leq j \leq m} .
$$

Write $P_{\psi} G^{*} e_{\infty}=\sum_{j=1}^{m} \gamma_{j} \psi_{j}=G^{*} \sum_{j=1}^{m} \gamma_{j} \theta_{j}$ and note that as $G$ has a dense range $G^{*}$ has null space $\{0\}$. Hence

$$
e_{\infty}=\sum_{j=1}^{m} \gamma_{j} \theta_{j}
$$

and $e_{\infty}=P_{\theta} e_{\infty}$ where $P_{\theta}$ is the orthogonal projection onto the $m$-dimensional subspace spanned by $\left\{\theta_{j}\right\}_{1 \leq j \leq m}$. Consequently

$$
\gamma=\left[\gamma_{1}, \ldots, \gamma_{m}\right]^{T}=M_{\theta}^{-1} \beta_{\theta}\left(e_{\infty}\right)
$$

To complete the proof, it remains to show that $\beta_{\theta}\left(e_{\infty}\right)=$ $\beta_{\theta}\left(e_{0}\right)$, where the first step is to rewrite the constraints $<u_{k+1}-u_{k}, \psi_{j}>=0,1 \leq j \leq m, k \geq 0$, in the form $<e_{k+1}-e_{k}, \theta_{j}>=0,1 \leq j \leq m, k \geq 0$. Equivalently, $<e_{k+1}-e_{0}, \theta_{j}>=0,1 \leq j \leq m, k \geq 0$, and taking the 


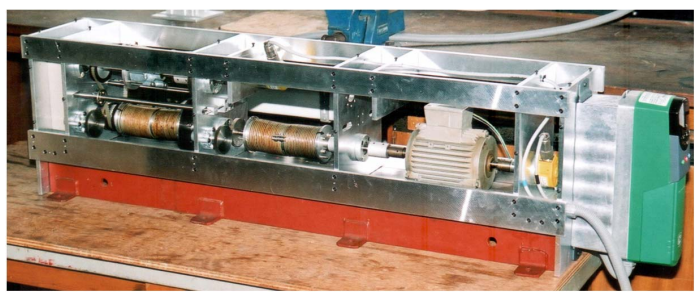

Fig. 3. Experimental benchmarking test facility.

limit, the condition follows from $<e_{\infty}, \theta_{j}>=<e_{0}, \theta_{j}>$, $1 \leq j \leq m$, and the definition of $\beta_{\theta}(e)$.

Finally, the norm $\left\|e_{\infty}\right\|^{2}$ is

$$
\begin{aligned}
\left\|e_{\infty}\right\|^{2} & =\left(M_{\theta}^{-1} \beta_{\theta}\left(e_{0}\right)\right)^{T} M_{\theta}\left(M_{\theta}^{-1} \beta_{\theta}\left(e_{0}\right)\right) \\
& =\beta_{\theta}\left(e_{0}\right)^{T} M_{\theta}^{-1} \beta_{\theta}\left(e_{0}\right) .
\end{aligned}
$$

Theorem 4 is a precise description of properties of the constrained NOILC model. For a single-input, single-output stable linear NMP system with zeros $z_{j}, 1 \leq j \leq m$, in the right-half plane, and $z_{j} \alpha \gg 0,1 \leq j \leq m$, convergence takes the form of initial reductions in error norm in $\mathcal{E}_{+}^{\perp}$ followed by a plateau where error norms in $\mathcal{E}_{+}$reduce infinitesimally from trial-to-trial. The model of the effect of these zeros as linear equality constraints on the dynamics of NOILC characterizes and predicts the error $e_{\infty}$ on the plateau component using (40)-(42).

In cases, such as the electromechanical NMP physical system of Section V, when the plant has only one right-half plane zero $z$, (40)-(42) give

$$
\begin{aligned}
e_{\infty} & =e^{-z t} \frac{2 z}{1-e^{-2 z \alpha}} \int_{0}^{\alpha} e^{-z t} e_{0}(t) d t \\
\left\|e_{\infty}\right\|^{2} & =\frac{2 z}{1-e^{-2 z \alpha}}\left[\int_{0}^{\alpha} e^{-z t} e_{0}(t) d t\right]^{2}
\end{aligned}
$$

clarifying the interaction between the basis function $e^{-z t}$ and $e_{0}$.

The error arising on the plateau is small only when $\beta_{\theta}\left(e_{0}\right)$ is small. This can be achieved only under the following two conditions: 1) the initial tracking error signal $e_{0}$ is small in norm and 2) the quantities $<\theta_{j}, e_{0}>, 1 \leq j \leq m$ are all small, which can be true if $e_{0}$ is not small but has this property in some time interval $\left[0, \alpha_{0}\right] \subset[0, \alpha]$ with $\operatorname{Re}\left[z_{j}\right] \alpha_{0} \gg 1,1 \leq$ $j \leq m$. This could be achieved naturally if, for example, the plant has zero initial conditions and the reference $r$ is zero in $\left[0, \alpha_{0}\right]$. Choosing $u_{0}(t)$ to be zero in this interval achieves the objective with no effort.

\section{EXPERIMENTAL VERIFICATION}

In this section, the electromechanical, NMP test facility shown in Fig. 3 is used to assess the validity of the methods and results of this brief. This facility is previously used to evaluate the performance of a wide variety of ILC schemes.

The system consists of a rotary mechanical system of inertias, dampers, torsional springs, a timing belt, pulleys, and gears. The NMP characteristic is achieved using the arrangement shown in Fig. 4, and a further spring-massdamper system is connected to the input to increase the relative degree and complexity. A 1000 pulse/rev encoder records the

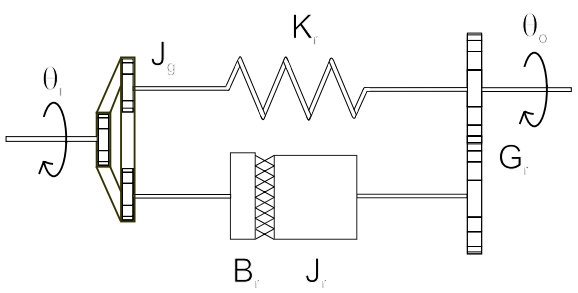

Fig. 4. Nonminimum phase characteristic, where $\theta_{i}$ and $\theta_{o}$ are the input and output positions, $J_{r}$ and $J_{g}$ are inertias, $B_{r}$ is a damper, $K_{r}$ is a spring, and $G_{r}$ represents the gearing.
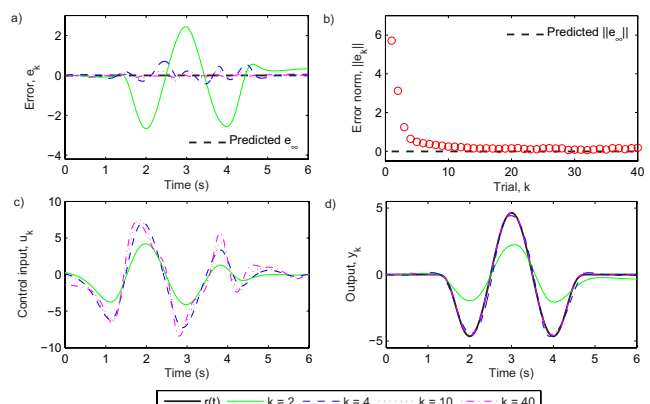

Fig. 5. Experimental results with initial zero period. (a) Error, $e_{k}$. (b) Error norm, $\left\|e_{k}\right\|$. (c) Control input, $u_{k}$. (d) Output, $y_{k}$.

output shaft position and a standard squirrel cage induction motor supplied by an inverter, operating in Variable Voltage Variable Frequency mode drives the load. The control scheme is implemented using a dSPACE ds1104 real-time board.

Frequency response test measurements with zero initial conditions are used to obtain the continuous-time plant transfer-function

$$
G(s)=\frac{165.95(4-s)}{s^{4}+21.5 s^{3}+170.28 s^{2}+368.52 s+663.82}
$$

which includes a proportional plus integral plus derivative stabilizer. The input and output are hence both shaft position, measured in radians. This system is NMP with one zero at $s=4$ (giving $z_{1}=4$ ) in the right-half of the complex plane. The 1-D subspace $\mathcal{E}_{+}$is therefore spanned by the single vector $e^{-4 t}$. Choosing a time interval $t \in[0,6]$, the factor $\alpha z_{1}=4 \times 6=24$ and hence $e^{-\alpha z_{1}}=e^{-24}$ is extremely small, and it is expected that the theoretical results of this brief should apply with good accuracy.

To show the close agreement existing between predicted and measured results, 40 trials of NOILC, using cost function weights $Q=5$, and $R=1$, and a sampling frequency of $250 \mathrm{~Hz}$, are performed, using the reference, $r(t)$, shown in Fig. 5(d). The reference comprises a sinusoidal positional movement (in radians), which is both preceded and followed by periods where it is set at zero. As stated in Section IV, the initial period in which $r(t)$ is zero means that $e_{0}$ will be small in this interval, and this leads to a low predicted final error, $e_{\infty}$, and associated norm $\left\|e_{\infty}\right\|$. These predicted values are shown in Fig. 5(a) and (b), respectively, and accurately match the experimental results.

To examine a case where a significant nonzero final error is predicted, the reference is now replaced with that shown in Fig. 6(d). This corresponds to the same movement as the previous reference; however, the starting point 


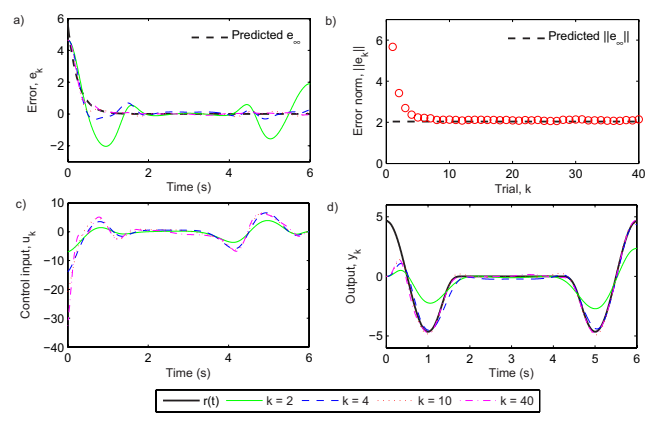

Fig. 6. Experimental results without initial zero period. (a) Error, $e_{k}$. (b) Error norm, $\left\|e_{k}\right\|$. (c) Control input, $u_{k}$. (d) Output, $y_{k}$.

is now changed to half way half-way through its duration. The initial period of $r(t)$ is now not zero so that $e_{0}$ will not be small in this interval, leading to predicted error values significantly different from zero, as shown in Fig. 6 (a) and (b). Again, 40 trials of NOILC, using the same cost function weights are performed, and the experimental error results, $e_{k}$ and $\left\|e_{k}\right\|$ obtained can be seen to closely match their predicted counterparts $e_{\infty}$ and $\left\|e_{\infty}\right\|$.

The results demonstrate the clear application of the analysis framework for design. The reference, initial input, and plant state must be selected such that the error norm is small over an initial time interval. The simplest way of achieving this is to select all three to be zero.

\section{CONCLUSION}

This brief modeled observed behavior of NOILC applied to NMP plants with $m \geq 1$ zeros. This model was in the form of an NOILC algorithm with equality constraints (orthogonality conditions) and was used to predict the values of error and error norm associated with the plateau using Lagrangian methods. The model explained the effect of the initial error $e_{0}$ and reference signal on the algorithm behavior and suggested that a small initial error and/or a reference signal with an initial period of inactivity are simple practical ways of improving performance and accuracy. Finally, model predictions were shown to have practical validity using an electromechanical benchmarking experiment.

In this brief, single-input, single-output systems were considered, where this class included many of the ILC algorithms that saw experimental verification. An immediate area for further control theory research was multiple-input, multipleoutput systems. For the former case, further research is required to consider the performance effects of NMP zeros in the presence of disturbances and uncertainty. The extension of these results to cases where a term $d_{0}(t) \in L_{2}[0, \alpha]$ representing a known trial-independent disturbance was added to (7) defining the operator $G$ is straightforward. Also longterm performance should be examined and also experimental performance for other reference signals. Extensions to noise disturbance and robustness considerations required considerable further research effort. The focus of this brief was to explain and quantify, with experimentally generated supporting results, the effects of NMP zeros on NOILC algorithm trial-totrial error convergence. Such zeros will affect the performance of all linear model-based ILC algorithms, and the results in this brief should play a critical role in further research aimed at modifying model-based ILC design to achieve the best possible performance in the presence of NMP zeros in the system considered.

\section{REFERENCES}

[1] D. A. Bristow, M. Tharayil, and A. G. Alleyne, "A survey of iterative learning control: A learning-based method for high-performance tracking control," IEEE Control Syst. Mag., vol. 26, no. 3, pp. 96-114, Jan. 2006.

[2] H.-S. Ahn, Y. Chen, and K. L. Moore, "Iterative learning control: Brief survey and categorization," IEEE Trans. Syst., Man, Cybern., C, Appl. Rev., vol. 37, no. 6, pp. 1109-1121, Nov. 2007.

[3] K. L. Barton and A. G. Alleyene, "A norm optimal approach to timevarying ILC with application to a multi-axis robotic testbed," IEEE Trans. Control. Syst. Technol., vol. 19, no. 1, pp. 166-180, Jan. 2011.

[4] D. Schindele and H. Aschemann, "ILC for a fast linear axis driven by pneumatic muscle actuators," in Proc. IEEE Int. Conf. Mechatronics, Apr. 2011, pp. 967-972.

[5] D. A. Bristow, "Weighting matrix design for robust monotonic convergence in norm optimal iterative learning control," in Proc. Amer. Control Conf., 2008, pp. 4554-4560.

[6] J. Ghosh and P. Paden, "Pseudo-Inverse based iterative learning control for linear nonminimum phase plants with unmodeled dynamics," ASME J. Dynamic Syst., Meas. Control, vol. 126, pp. 661-665, 2001.

[7] K. Kinosita, T. Sogo, and N. Adachi, "Iterative learning control using adjoint systems and stable inversion," Asian J. Control, vol. 4, no. 1, pp. 60-67, 2002.

[8] D. H. Owens and B. Chu, "Modelling of non-minimum-phase effects in discrete-time norm-optimal iterative learning control,' Int. J. Control, vol. 83, no. 10, pp. 2012-2027, 2010.

[9] C. T. Freeman, E. Rogers, A.-M. Hughes, J. H. Burridge, and K. L. Meadmore, "Iterative learning control in healthcare: Electrical stimulation and robotic-assisted upper limb stroke rehabilitation," IEEE Contr. Syst. Mag., vol. 32, no. 1, pp. 18-43, Jul. 2012.

[10] N. Amann, D. H. Owens, and E. Rogers, "Iterative learning control using optimal feedback and feedforward actions," Int. J. Control, vol. 65, no. 2 , pp. 277-293, 1996.

[11] P. R. Halmos, A Hilbert Space Problem Book. New York, NY, USA: Springer-Verlag, 1982.

[12] E. Rogers, D. H. Owens, H. Werner, C. T. Freeman, P. L. Lewin, S. Kichhoff, C. Schmidt, and G. Lichtenberg, "Norm optimal iterative learning control with application to problems in accelerator based free electron lasers and rehabilitation robotics," Eur. J. Control, vol. 16, no. 5, pp. 497-524, 2010.

[13] J. Dieudonné, Foundations of Modern Analysis. San Diego, CA, USA: Academic Press, 1969. 